\title{
ROBERT FAGES
}

MICHEL GANGNET

\section{Sur les modèles probabilistes de prévision des localisations et des déplacements dans une agglomération}

Revue française d'automatique, d'informatique et de recherche opérationnelle. Recherche opérationnelle, tome 11, $\mathrm{n}^{\mathrm{o}} 3$ (1977), p. 281-303.

<http://www.numdam.org/item?id=RO_1977_11_3_281_0>

(C) AFCET, 1977, tous droits réservés.

L'accès aux archives de la revue « Revue française d'automatique, d'informatique et de recherche opérationnelle. Recherche opérationnelle » implique l'accord avec les conditions générales d'utilisation (http://www.numdam.org/ legal.php). Toute utilisation commerciale ou impression systématique est constitutive d'une infraction pénale. Toute copie ou impression de ce fichier doit contenir la présente mention de copyright.

\section{Numdam}

Article numérisé dans le cadre du programme

Numérisation de documents anciens mathématiques

http://www.numdam.org/ 


\title{
SUR LES MODĖLES PROBABILISTES DE PRÉVISION DES LOCALISATIONS ET DES DÉPLACEMENTS DANS UNE AGGLOMÉRATION $\left({ }^{1}\right)$
}

\author{
par Robert Fages $\left({ }^{2}\right)$ et Michel Gangnet $\left({ }^{3}\right)$
}

\begin{abstract}
Résumé. - L'article reprend la construction du modèle exponentiel de transport entre les zones d'une agglomération et propose diverses extensions aux travaux d'A. Wilson. L'usage de l'approximation de Stirling est évité ce qui justifie le modèle pour toutes les valeurs de flux interzones. Dans le calcul de la fonction de coût, le paramètre est traité systématiquement comme une inconnue et non ajusté aux observations. Cette dernière attitude revenant en fait à l'utilisation d'un modele gravitaire.

Une notion de la sensibilité d'une population aux coûts est proposée. L'article définit sous des hypothèses explicites une méthode permettant l'obtention d'une matrice de coût à partir d'un trafic observé. D'autre part, une nouvelle preuve des relations du modèle avec le problème de Hitchcock est donnée. Diverses généralisations sont abordées, notamment la multiplicité des modes de transport et la non-uniformité du comportement des individus.
\end{abstract}

\section{POSITION DU PROBLĖME}

Considérons une ville ou une région dont on a fait une partition en un ensemble $Z$ de $n$ zones $Z=\left\{Z_{i}, i=1, n\right\}$, les zones doivent satisfaire des conditions de régularité que nous n'examinerons pas ici ( $c f$. Broadbent, 1969). Soit $I$ un ensemble d'individus, de ménages ou d'activités; Card $(I)=N$. On rencontre en modélisation urbaine les deux types de problèmes suivants:

- Problèmes de localisation: il s'agit de prévoir une localisation $L=\left(l_{1}, l_{2}, \ldots, l_{n}\right)$, où $l_{i}$ est le nombre des éléments de $I$ localisés en zone $Z_{i}$ avec

$$
\text { C.L. } \mid \sum_{i=1}^{n} l_{i}=N \quad, \quad l_{i} \geqslant 0 \text { pour tout } i
$$

sous certaines conditions dites conditions de comportement. Par excmple prévision du nombre de ménages résidant en $Z_{i}$ connaissant le coût $c_{i}$ de cette localisation et la moyenne $\bar{c}=\frac{1}{N} \sum_{i} l_{i} c_{i}$. Voir 2 .

\footnotetext{
( $\left.{ }^{1}\right)$ Reçu fév. 1976.

$\left({ }^{2}\right)$ Université Claude Bernard Lyon I, Villeurbanne.

$\left({ }^{3}\right)$ Unité Pédagogique d'Architecture de Lyon, Lyon.
}

R.A.I.R.O. Recherche Opérationnelle/Operations Research, vol. 11, n 3, août 1977. 
- Problèmes de trafic: on s'intéresse aux relations inter-zones il s'agit de prévoir un trafic $T=t_{i j}$ où $t_{i j}$ est le flux entre les zones $Z_{i}$ et $Z_{i}$ avèc

$$
\text { C.T. } \mid \begin{aligned}
& \sum_{j} t_{i j}=O_{i} \quad \sum_{i} t_{i j}=D_{j} \quad t_{i j} \geqslant 0 \text { pour tout } i, j \\
& \sum_{i} O_{i}=\sum_{j} D_{j}=\sum_{i j} t_{i j}=N
\end{aligned}
$$

sous des conditions de comportement. $O_{i}$ et $D_{j}$ représentent les quantités fixées d'émission et de réception des zones $Z_{i}$ et $Z_{j}$. On les supposera strictement positifs.

Par exemple prévision du nombre d'actifs travaillant en $Z_{j}$ et habitant en $Z_{i}$ connaissant la matrice des coûts de déplacement $\left[c_{i j}\right]$ et une borne supérieure de l'espérance mathématique de ceux-ci pour tous les individus.

Dans cette situation très générale deux méthodes ont été proposées par Wilson $(1970,1974)$ :

- recherche sous certaines hypothèses de la distribution la plus probable

- maximisation d'une entropie.

\subsection{Recherche de la distribution la plus probable}

\subsection{Probabilité d'une localisation (resp. d'un trafic)}

Les conditions de comportement sont des hypothèses sur la façon dont les éléments de $I$ effectuent le choix de leur zone de localisation $Z_{i}$ (resp. des zones $Z_{i}$ et $Z_{j}$ ). Sous une hypothèse générale concernant les conditions de comportement on peut caractériser la localisation (resp. le trafic) de plus grande probabilité et la construire numériquement dans les cas les plus simples.

Cas d'une localisation: Soit $g_{L}$ l'application de $Z$ dans $\mathscr{P}(I)$ où $g_{L}\left(Z_{i}\right)$ est la liste non ordonnée des individus localisés en $Z_{i}$. On a $l_{i}=$ Card $\left(g_{L}\left(Z_{i}\right)\right)$. A chaque application $g_{L}$ est attaché une localisation $L$ notée $L\left(g_{L}\right)$.

Cas d'un trafic: Soit $g_{T}$ l'application de $Z \times Z$ dans $\mathscr{P}(I)$ où $g_{T}\left(Z_{i}, Z_{j}\right)$ est la liste non ordonnée des individus allant de $Z_{i}$ à $Z_{j}$. On a $t_{i j}=$ Card $\left(g_{T}\left(Z_{i}, Z_{j}\right)\right)$. A chaque application $g_{T}$ est attaché un trafic $T$ noté $T\left(g_{T}\right)$.

Hypothèse: Les conditions de comportement ne sont pas nominatives et peuvent être exprimées collectivement comme conditions sur $L$ (resp. $T$ ).

Autrement dit et dans cette première formulation du problème cela implique que tous les éléments de $I$ ont le même comportement.

DÉfinition: Une localisation $L$ (resp. un trafic $T$ ) sera dit réalisable si $L$ (resp. $T$ ) vérifie $C L$ (resp. $C T$ ) et les conditions de comportement. 
DÉFINITION: On appelle état de localisation (resp. état de trafic) toute application $g_{L}$ (resp. $\left.g_{T}\right)$ telle que $L\left(g_{L}\right)$ (resp. $T\left(g_{T}\right)$ ) soit réalisable.

Conséquence: Les états de localisation (resp. les états de trafic) sont équiprobables. En effet du point de vue des conditions de comportement, de l'hypothèse et des contraintes $C L$ (resp. $C T$ ) rien ne distingue deux états de localisation (resp. de trafic).

A partir d'ici nous allons poursuivre l'exposé sur le seul cas des problèmes de trafic; les résultats sont analogues dans le cas plus simple des localisations.

Si $E$ est l'ensemble fini de tous les états de trafic, il s'ensuit en utilisant la mesure dénombrement que la probabilité de réalisation d'un trafic $T$ est :

$$
P(T)=\frac{\operatorname{Card}\left(g_{T}: T=T\left(g_{T}\right)\right)}{\text { Card }(E)}
$$

Le numérateur de $P(T)$ est le nombre de rangements discernables de $N$ objets en $n^{2}$ cases de capacité $t_{i j}$ d'où :

$$
P(T)=\frac{N !}{\prod_{i j}\left(t_{i j}\right) !} \frac{1}{\operatorname{Card}(E)}
$$

Card $(E)$ est indépendant des inconnues $\left[t_{i j}\right]$ et ne dépend que des contraintes C.T. et des conditions de comportement. Par exemple s'il n'y a pas de conditions de comportement on aura :

$$
\text { Card }(E)=\frac{(N !)^{2}}{\prod_{i} O_{i} ! D_{i} !}
$$

\subsection{Recherche du trafic le plus probable}

Le trafic de plus grande probabilité sera considéré comme un équilibre statistique autour duquel on observera des fluctuations. Pour le problème de prévision qui nous préoccupe on se ramènera à la détermination du trafic le plus probable à l'exemple de la mécanique statistique ( $c f$. Wilson, 1970).

Ainsi chercher $T=\left[t_{i j}\right]$ tel que $P(T)$ soit maximum est équivalent à déterminer les $t_{i j}$ tels que $W=\frac{N !}{\prod_{i j}\left(t_{i j} !\right)}$ soit maximum; c'est-à-dire le trafic réalisable qui admet le nombre maximum d'états de trafic associés. Ici intervient de façon essentielle l'équiprobabilité des états de trafic. Il est aussi équivalent de rechercher le maximum de $H=\log W$. Ainsi le trafic réalisable le plus probable est celui qui maximise $H$.

Les inconnues $t_{i j}$ sont des entiers mais dans le but d'obtenir une solution analytique nous définirons $H$ pour des $t_{i j}$ réels par

$$
H(T)=\log (N !)-\sum_{i j} \log \left(\Gamma\left(t_{i j}+1\right)\right), t_{i j} \geqslant 0
$$

où $\Gamma$ est la fonction gamma d'Euler. 
$H$ est fonction strictement concave des $\left[t_{i j}\right]$, en effet :

$$
\frac{d^{2} \log \Gamma(x+1)}{d x^{2}}=\sum_{i=1}^{+\infty} \frac{1}{(i+x)^{2}}>0 \quad \text { pour } \quad x \geqslant 0
$$

Donc, sous la seule hypothèse que les contraintes (état réalisable) du problème de maximisation exposé ci-avant soient linéaires en $\left[t_{i j}\right]$ la solution du problème continu sera voisine de la solution du problème discret.

1.13. Calcul des dérivées partielles de $H$ série.

On a $\frac{\partial H}{\partial t_{i j}}=-\frac{\partial}{\partial t_{i j}} \log \left(\Gamma\left(t_{i j}+1\right)\right)$ dont le calcul exact est donné par une

Rappelons le résultat classique suivant :

Proposition 1: Soit $f$ une fonction réelle convexe de classe $C^{1}$, alors pour tout $x_{1}, x_{2}$ dans $\mathbf{R}$ on $a$ :

$$
f\left(x_{2}\right) \geqslant f\left(x_{1}\right)+f^{\prime}\left(x_{1}\right)\left(x_{2}-x_{1}\right)
$$

si de plus $f$ est strictement convexe alors:

$$
f\left(x_{2}\right)>f\left(x_{1}\right)+f^{\prime}\left(x_{1}\right)\left(x_{2}-x_{1}\right)
$$

Montrons alors la :

Proposition 2: Soit $f$ une fonction réelle strictement convexe de classe $C^{1}$ dans $\mathbf{R}$ alors : pour tout $x \in \mathbf{R}$, pour tout $\Delta \in \mathbf{R}, \Delta \neq 0$, il existe $\theta(x)$ unique, $\theta \in] 0,1[$ tel que :

$$
f(x+\Delta \theta(x))-f(x-(1-\theta(x)) \Delta)=f^{\prime}(x) \Delta
$$

Démonstration : Étudions la fonction

on a :

$$
\begin{aligned}
& g(\theta)=f(x+\Delta \theta)-f(x-(1-\theta) \Delta)-f^{\prime}(x) \Delta \\
& g(0)=f(x)-f(x-\Delta)-f^{\prime}(x) \Delta<0 \\
& g(1)=f(x+\Delta)-f(x)-f^{\prime}(x) \Delta>0
\end{aligned}
$$

d'après (1).

$g$ est fonction de classe $C^{1}$ de $\theta$ et

$$
g^{\prime}(\theta)=\left[f^{\prime}(x+\Delta \theta)-f^{\prime}(x-(1-\theta) \Delta)\right] \Delta>0
$$

car $f$ étant strictement convexe, $f^{\prime}$ est strictement croissante.

Ainsi $g(\theta)$ est strictement croissante sur $[0,1]$ et d'après .(3) et (4) elle admet un zéro unique sur $] 0,1[$; soit $\theta(x)$ ce zéro, d'où (2).

Application : $\log \Gamma(x+1)$ est on l'a vu strictement convexe de classe $C^{\infty}$ pour $x \in[0,+\infty[$; utilisons la proposition 2 avec $\Delta=1$. On constatera que celle-ci est encore valable pour $x=0$. 
Il existe $\theta(x) \in] 0,1[$ unique tel que

$$
\log \Gamma(x+1+\theta(x))-\log \Gamma(x+\theta(x))=\frac{d \log \Gamma(x+1)}{d x}
$$

soit

$$
\frac{d \log \Gamma(x+1)}{d x}=\log (x+\theta(x))
$$

Ainsi : $\quad \frac{\partial H}{\partial t_{i j}}=-\log \left(t_{i j}+\theta\left(t_{i j}\right)\right) \quad 0<\theta\left(t_{i j}\right)<1$

pour $t_{i j} \in[0,+\infty[$.

Cette démonstration évite l'utilisation comme le font certains auteurs de la formule d'approximation de Stirling qui rend les calculs incertains pour

$$
t_{i j} \leqslant 15
$$

Dans le cas de la localisation on a de même:

$$
\frac{\partial H}{\partial l_{i}}=-\log \left(l_{i}+\theta\left(l_{i}\right)\right) \quad 0<\theta\left(l_{i}\right)<1
$$

pour $l_{i} \in[0,+\infty[$.

\subsection{Maximisation de l'entropie}

Considérons le cas des problèmes de localisation. Chaque élément de $I$ possède un $\mathrm{n}^{\circ}$ de zone $i$ qui peut prendre les valeurs $1, n$. Soit $X$ la variable aléatoire discrète associée à l'épreuve : tirage avec remise dans une urne finie contenant les $N$ éléments de $I$. Le problème est de trouver les $P_{i}=P(X=i)$, $\sum_{i} p_{i}=\sum_{i} \frac{l_{i}}{N}=1$ sous certaines conditions.

Dès que ces conditions s'expriment sous la forme

$$
E\left(f_{j}(X)\right)=\overline{f_{j}} \quad 1 \leqslant j \leqslant r
$$

où les $\bar{f}_{j}$ sont connues ainsi que les valeurs de $f_{j}(i)$ pour tout $i$ et pour tout $j$, Wilson (1970) propose en suivant Jaynes (1968) et Tribus (1969) de chercher les $p_{i}$ qui maximisent l'entropie $S=-\sum_{i} p_{i} \log p_{i}$ sous les conditions (7). Les conditions (7) jouent le rôle des conditions de comportement de la formulation précédente et la recherche du maximum se fait en utilisant la méthode des multiplicateurs de Lagrange. Les calculs sont analogues dans le cas des problèmes de trafic.

\subsection{Comparaison des bases théoriques des deux méthodes}

Les deux méthodes que nous venons d'exposer conduisent aux mêmes résultats. En effet, en écrivant (5) pour les $t_{i j}$ entiers voisins des $t_{i j}+\theta\left(t_{i j}\right)$ on trouve les mêmes dérivées partielles pour $H$ et l'entropie

$$
\frac{\partial H}{\partial t_{i j}} \simeq-\log t_{i j}=\frac{\partial}{\partial t_{i j}}\left[-\sum_{i j}\left(t_{i j} \log t_{i j}-t_{i j}\right)\right]
$$

vol. $11, \mathrm{n}^{\circ} 3$, août 1977 
soit

$$
\frac{\partial H}{\partial t_{i j}} \simeq \frac{\partial}{\partial t_{i j}}\left[-\sum_{i j} t_{i j} \log t_{i j}\right]
$$

$\operatorname{car} \sum_{i j} t_{i j}=N$ est constant.

Wilson (1970) soutient cependant que du point de vue théorique la seconde doit être préférée à la première. Nous venons de montrer que l'utilisation de la formule d'approximation de Stirling dans la première méthode était inutile, ce qui fait que la validité de la recherche de la distribution la plus probable tient toute entière dans l'hypothèse énoncée plus haut (1.11). La méthode de la maximisation de l'entropie en plus du fait qu'elle utilise la quantité d'information de Shannon, ( $c f$. March, 1975), ne repose à notre connaissance sur aucun théorème. On peut à ce sujet citer Lindley (1972): “An ingenious principle for the use of information in developing "ignorance" prior distributions has been put forward by Jaynes (1968) and expounded by Tribus (1969). According to the latter, this consists in choosing that distribution which maximizes the entropy subject to constraints supplied by the given information. Tribus's stimulating but mathematically infuriating book makes out a good case." Cela dit les présomptions en faveur de cette méthode sont fortes comme le montrent par exemple les excellents résultats obtenus par Bussière (1972) dans le cas continu.

\section{REMARQUES SUR LE PROBLÈME DE LOCALISATION}

Considérons le problème suivant (on pose $p_{i}=\frac{l_{i}}{N}$ )

$$
P L \mid \begin{array}{ll}
S=-\sum_{i} p_{i} \log p_{i} & \text { Maximum } \\
p_{i} \geqslant 0 \sum_{i} p_{i}=1 & \text { C.L. } \\
\sum_{i} p_{i} c_{i}=\bar{c} & \text { condition de comportement }
\end{array}
$$

L'équivalence montrée en 1.3 nous autorise à maximiser $S$ au lieu de $H$.

La solution de ce problème est donnée par (méthode des multiplicateurs de Lagrange) le système

$$
S L \mid \begin{aligned}
& p_{i}=\exp \left(-\alpha-\beta c_{i}\right) \\
& \text { avec } \\
& \alpha=\log \left(\sum_{i=1}^{n} \exp \left(-\beta c_{i}\right)\right) \\
& \text { et } \\
& \sum_{i=1}^{n} \exp \left(-\beta c_{i}\right)\left(c_{i}-\bar{c}\right)=0
\end{aligned}
$$

R.A.I.R.O. Recherche Opérationnelle/Operations Research 
On peut noter que si les $c_{i}$ et $\bar{c}$ sont des entiers et en posant $\exp (-\beta)=X$, l'existence de $\beta$ est assurée par un théorème figurant, sans preuve, dans la géométrie de Descartes de 1637.

L'équation (8) est sans solution si $\bar{c}>\operatorname{Max}_{i} c_{i}$ ou si $\bar{c}<\operatorname{Min}_{i} c_{i}$, les exponentielles étant $>0$ pour $\beta$ fini.

Si l'on cherche le maximum de $S$ sans la condition de comportement alors

$$
\beta=0, \quad \sum_{i} c_{i}=N \bar{c}, \alpha=\log n \quad \text { et } \quad p_{i}=\frac{1}{n} .
$$

Dans le système $S L$ les inconnues sont $\alpha$ et $\beta$; (8) définit $\beta$ comme fonction implicite de $\bar{c}$ et il est intéressant d'étudier certaines valeurs en fonction de $\beta$.

Étudions la limite des $p_{i}$ quand $\beta \rightarrow \pm \infty$. On a :

$$
p_{i}=\frac{1}{1+\sum_{\substack{j \\ i \neq j}}\left(\frac{e^{c_{i}}}{e^{c_{j}}}\right)^{\beta}}
$$

On aura donc :

et

$$
\left\{\begin{array}{l}
\lim _{\beta \rightarrow+\infty} p_{i}=0 \quad \text { si } \quad c_{i}>\operatorname{Min}_{i} c_{i} \\
\lim _{\beta \rightarrow+\infty} p_{i}=\frac{1}{k} \quad \text { avec } \quad k=\operatorname{Card}\left(i \mid c_{i}=\operatorname{Min}_{i} c_{i}\right)
\end{array}\right.
$$

$$
\left\{\begin{array}{l}
\lim _{\beta \rightarrow-\infty} p_{i}=0 \quad \text { si } \quad c_{i}<\operatorname{Max}_{i} c_{i} \\
\lim _{\beta \rightarrow-\infty} p_{i}=\frac{1}{k} \quad \text { avec } \quad k=\operatorname{Card}\left(i \mid c_{i}=\operatorname{Max}_{i} c_{i}\right)
\end{array}\right.
$$

On retrouve les solutions des problèmes (triviaux) de programmation linéaire suivants :

$$
\begin{array}{l|l|l}
P L_{\min } & \sum_{i} p_{i}=1 \quad p_{i} \geqslant 0 \\
\sum_{i} p_{i} c_{i} \text { Minimum } & P L_{\max } & \mid \begin{array}{l}
\sum_{i} p_{i}=1 \quad p_{i} \geqslant 0 \\
\sum_{i} p_{i} c_{i}
\end{array} \text { Maximum }
\end{array}
$$

On a $S=\alpha+\beta \bar{c}$ ce qui définit $S$ comme fonction de $\beta$ ou de $\bar{c}$. $\beta$ et $\bar{c}$ ne sont pas indépendants en raison de (8).

Calculons $\frac{d S}{d \beta}: \quad S=\log \sum_{i} e^{-\beta c i}+\beta \sum_{i} \frac{e^{-\beta c i}}{\sum_{i} e^{-\beta c i}} c_{i}$

d'où en posant $e^{-\beta c i}=e_{i}$ et $\sum_{i} e_{i}=e$.

$$
\frac{d S}{d \beta}=\beta\left[\frac{\left(\sum_{i} e_{i} c_{i}\right)^{2}}{e^{2}}-\frac{\sum_{i} e_{i} c_{i}^{2}}{e_{.}}\right]=-\beta \operatorname{Var}(c)
$$

vol. $11, \mathrm{n}^{\circ} 3$, août 1977 
D'autre part en différenciant (8) on a :

$$
\sum_{i} e_{i} c_{i}\left(c_{i}-\bar{c}\right) \frac{d \beta}{d \bar{c}}+e_{.}=0
$$

d'où

$$
\frac{d \beta}{d \bar{c}}=-\frac{1}{\operatorname{Var}(c)} \quad \text { Soit } \quad \frac{d \bar{c}}{d \beta}=-\operatorname{Var}(c)
$$

et

$$
\frac{d S}{d \bar{c}}=\dot{\beta}
$$

Ces relations permettent l'étude des fonctions $S=\varphi(\beta)=\psi(\bar{c})$ et de $\beta=f(\bar{c})($ fig. 1).

Dans les applications on trouve toujours $\beta>0$ ce qui correspond à $c_{\min }<\bar{c}<\frac{1}{n} \sum_{i} c_{i}$; en effet on doit s'attendre à ce que $p_{i}$ soit fonction décroissante des $c_{i}$.

Figure 1 .

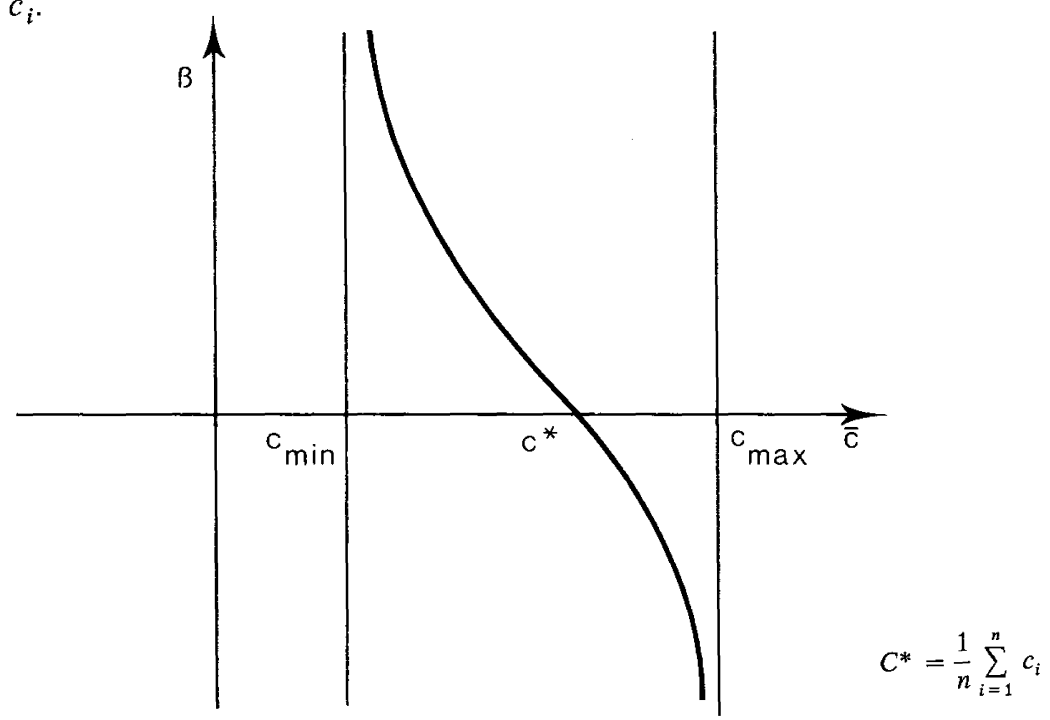

\section{SUR LE PROBLÈME DU TRAFIC}

\section{1. Matrice de coût unique}

On se propose de prévoir les déplacements $\left[t_{i j}\right]$ entre les zones $Z_{i}$ et $Z_{j}$ d'effectifs $O_{i}$ et $D_{j}$ connus, par exemple relations domicile-travail. On suppose qu'il n'existe qu'un seul moyen de transport et donc une seule matrice de coût $\left[c_{i j}\right]$.

Choisissons comme condition de comportement des habitants de la ville

$$
\sum_{i j} t_{i j} c_{i j} \leqslant N \bar{c}
$$

R.A.I.R.O. Recherche Opérationnelle/Operations Research 
Cette condition vérifie l'hypothèse générale du 1.11 et signifie que le coût moyen dépensé par l'ensemble des individus a une limite supérieure $\bar{c}$. Sachant que le trafic $T$ est réalisé, la probabilité de trouver un individu donné sur le trajet de $Z_{i}$ à $Z_{j}$ est $\frac{t_{i j}}{N}$, (9) exprime que l'espérance mathématique de dépense de cet individu est inférieure ou égale à $\bar{c}$.

3.11. Solution du problime

Le problème peut se formuler ainsi :

Chercher $T=\left[t_{i j}\right] \in S \cap D$ qui maximise la fonction strictement concave $H(T)$

où $\quad S$ est le domaine convexe défini par $t_{i j} \geqslant 0$

$P_{1}$ $D$ est le domaine convexe défini par

$$
\begin{aligned}
& \sum_{j} t_{i j}=O_{i} \quad, \sum_{i} t_{i j}=D_{j} \\
& \sum_{i, j} t_{i j} c_{i j}+p=N \bar{c}, \quad p \geqslant 0
\end{aligned}
$$

(10) est équivalente à la condition (9).

Considérons d'abord le problème

$P_{1}^{\prime} \mid$ chercher $T \in D$ qui maximise $H(T)$

Si $D$ est non vide la solution de $P_{1}^{\prime}$ vérifie les conditions nécessaires données par la méthode des multiplicateurs de Lagrange ( $c f .1 .13$ ).

$$
\frac{\partial H}{\partial t_{i j}}=-\log \left(t_{i j}+\theta\left(t_{i j}\right)\right)=\lambda_{i}+\mu_{j}+\beta c_{i j}
$$

avec de plus

$$
\frac{\partial H}{\partial P}=0=\beta \quad \text { si } \quad p \neq 0
$$

où les $\lambda_{i}, \mu_{j}$ et $\beta$ sont les multiplicateurs.

(11) entraîne $t_{i j}=a_{i} b_{j} e^{-\beta c_{i j}}-\theta\left(t_{i j}\right)$ où l'on a posé $a_{i}=e^{-\lambda_{i}}, b_{j}=e^{-\mu_{j}}$. Mais comme on s'intéressera à une valeur entière de $t_{i j}$ on peut déjà et à des fins de simplification d'écriture poser

$$
\left.t_{i j}=a_{i} b_{j} e^{-\beta c_{i j}} \quad \text { car } \theta\left(t_{i j}\right) \in\right] 0,1[.
$$

$I^{\text {er }}$ cas : La donnée $\bar{c}$ est suffisamment grande pour que $p>0$.

Dans ce cas on écrit (12) et

$$
t_{i j}=a_{i} b_{j}
$$

Mais $\sum_{j} t_{i j}=a_{i} \sum_{j} b_{j}=O_{i}$ où nous posons $\sum_{j} b_{j}=1$ car les conditions (C.T) bien que compatibles ne sont pas indépendantes (il reste un degré de liberté). 
alors $a_{i}=0_{i} ;$ mais $\sum_{i} t_{i j}=b_{j} \sum_{i} O_{i}=b_{i} N=D_{j}$

d'où

$$
T^{*}=\left[t_{i j}^{*}\right] \quad, \quad t_{i j}^{*}=\frac{O_{i} D_{j}}{N}
$$

et si nous posons

$$
c^{*}=\frac{1}{N} \sum_{i j} \frac{O_{i} D_{j}}{N} c_{i j}
$$

(13) est solution à une unité près par élément de $P_{1}^{\prime}$ (et de $P_{1}$ car $T^{*} \in S$ ) si $\bar{c} \geqslant c^{*}$.

$$
2^{\mathrm{e}} \text { cas }: \bar{c}<c^{*}
$$

Dans ce cas $p=0$ et $\beta \neq 0$ (le théorème de Kuhn et Tucker permet même d'affirmer $\beta>0$ ).

Les $t_{i j}$ solution de $P_{1}^{\prime}$ sont alors nécessairement solution du système:

$$
S_{1} \mid \begin{aligned}
& t_{i j}=a_{i} b_{j} e^{-\beta c_{i j}} \\
& \sum_{j} t_{i j}=O_{i} \quad, \quad \sum_{i} t_{i j}=D_{j} \\
& \sum_{i j} t_{i j} c_{i j}=N \bar{c}
\end{aligned}
$$

aux inconnues $t_{i j}, a_{i}, b_{j}$ et $\beta$.

Nous supposerons que $c_{i j}$ ne peut s'écrire sous la forme $p_{i}+q_{j}$

En effet dans le cas contraire la solution $T^{*} \mathrm{du} 1^{\text {er }}$ cas vérifie les premières équations de $S_{1}$ mais ne peut satisfaire à la dernière car $\bar{c}<c^{*}$. Le système $S_{1}$ serait alors incompatible et donc $D=\emptyset$ et $P_{1}$ n'a pas de solution.

La solution $T$ de $S_{1}$ a par construction ses éléments positifs donc $T \in S$ et la solution de $P_{1}$ vérifie nécessairement $S_{1}$. Réciproquement $S_{1}$ ne peut avoir, s'il en a, plusieurs solutions car $H$ est strictement concave et $D$ convexe. Autrement dit $S_{1}$ avec $P_{1}$ a soit une solution unique, soit pas de solution.

Considérons maintenant un autre problème :

$$
\begin{array}{l|l}
P_{2} \mid \underset{T \in S_{\cap \Delta}}{\operatorname{Min}}\left[F_{\alpha}(T)=\frac{1}{\alpha} \sum_{i j} t_{i j} \log t_{i j}+\sum_{i j} t_{i j} c_{i j}\right] \\
S: t_{i j} \geqslant 0 \\
\Delta: \sum_{j} t_{i j}=O_{i} \quad, \quad \sum_{i} t_{i j}=D_{j}
\end{array}
$$

où $\alpha>0$ est un paramètre fixé.

$F_{\alpha}(T)$ est strictement convexe, $\Delta$ est convexe, le raisonnement précédent montre que $P_{2}$ et

$$
P_{2}^{\prime} \mid \operatorname{Min}_{T \in \Delta} F_{\alpha}(T)
$$


ont avec

$$
\begin{array}{l|l}
S_{2} & \begin{array}{l}
t_{i j}=a_{i} b_{j} e^{-\alpha c_{i j}}=a_{i} s_{i j}^{(\alpha)} b_{j} \\
\sum_{j} t_{i j}=O_{i} \quad, \quad \sum_{i} t_{i j}=D_{j}
\end{array} \quad \text { où } \quad s_{i j}^{(\alpha)}=e^{-\alpha c_{i j}}
\end{array}
$$

la même solution.

Or Bacharach (1965) a montré qu'en particulier si $s_{i j}^{\alpha}>0$ pour tout $i, j$ (et c'est le cas ici) $S_{2}$ admet une solution unique donnée par la suite convergente :

$$
\mid \begin{aligned}
& b_{j}^{0}=1 \quad \forall j \\
& a_{i}^{n+1}=\frac{O_{i}}{\sum_{j} s_{i j}^{(\alpha)} b_{j}^{n}} \quad \forall i \\
& b_{j}^{n+1}=\frac{D_{j}}{\sum_{i} s_{i j}^{(\alpha)} a_{i}^{n+1}} \forall j
\end{aligned}
$$

$P_{2}$ admet donc quel que soit $\alpha$ une solution unique que d'ailleurs on sait construire numériquement.

Pour $T$ solution de $P_{2}$ construisons l'application

$$
C(\alpha)=\frac{1}{N} \sum_{i j} t_{i j} c_{i j} \text { de } \mathbf{R}^{+} \text {dans } \mathbf{R}
$$

il est clair que cette application est continue.

$$
a-\lim _{\alpha \rightarrow 0} C(\alpha)=c^{*} \quad \text { car } \quad \lim _{\alpha \rightarrow 0} s_{i j}^{(\alpha)}=1 \quad \text { pour tout } i, j .
$$

et (13) est solution de $S_{2}$ quand $\alpha \rightarrow+\infty$;

$$
b-\lim _{\alpha \rightarrow+\infty} C(\alpha)=c_{\min }=\frac{1}{N} \sum_{i j} \overline{t_{i j}} c_{i j}
$$

où $\bar{T}=\left[\bar{t}_{i j}\right]$ est solution du problème de programmation linéaire

$$
P T_{\min } \mid \underset{T \in S \cap S}{\operatorname{Min}} F(T)=\sum_{i j} \bar{t}_{i j} c_{i j}
$$

$P T_{\min }$ est un problème de transport (Problème de Hitchcock).

En effet $\lim _{\alpha+\infty} F_{\alpha}(T)=F(T)$ et quand $\alpha \rightarrow+\infty$ la solution de $P_{2}$ tend continuement vers une solution de $P T_{\min }$.

Ce résultat est démontré différemment par S. P. Evans (1973) qui caractérise notamment la limite lorsqu'il n'y a pas unicité de la solution de $\dot{P} T_{\min }$.

A. W. Evans (1971) quant à lui fait une étude détaillée de la fonction $C(\alpha)$ et montre que si la matrice des coûts $c_{i j}$ n'est pas dégénérée sous la forme $c_{i j}=p_{i}+q_{j}$, C est strictement décroissante. 

On démontrerait de même que $\lim _{\alpha \rightarrow-\infty} C(\alpha)=c_{\max }=\frac{1}{N} \sum_{i j} t_{i j} c_{i j}$ où $\underline{T}=\left[t_{\underline{i j}}\right]$
est solution de $P T_{\max }$

$$
P T_{\max } \mid \underset{\underline{T} \in S \cap \Delta}{\operatorname{Max}} F(\underline{T})
$$

avec naturellement $c_{\min } \leqslant c^{*} \leqslant c_{\max }$.

Nous avons notamment établi que $C(\alpha)$ est une bijection de $\mathbf{R}^{+}$sur ]$\left.c_{\min }, c^{*}\right]$.

Le problème $P_{2}$ n'a été évoqué ici que pour ce dernier résultat.

En effet on peut interpréter le système $S_{1}$ comme la recherche de $\beta \in \mathbf{R}^{+}$ tel que

$$
C(\beta)=\bar{c}
$$

or cela n'est possible que si $c_{\min }<\bar{c} \leqslant c^{*}$.

Donc si

$\bar{c}<c_{\min } \quad D=\emptyset$ et $S_{1}$ donc $P_{1}$ n'ont pas de solution.

$\bar{c}=c_{\min } \quad S_{1}$ admet une des solutions du problème de transport $P T_{\min }$. $c_{\min }<\bar{c}<c^{*} S_{1}$ admet une solution unique.

Un algorithme performant a été mis au point pour en construire numériquement la solution.

(Le texte du programme Fortran correspondant sera remis à tout lecteur qui en fera la demande aux auteurs.)

$\bar{c} \geqslant c^{*} \quad S_{1}$ admet la solution (13). Les coûts $c_{i j}$ n'interviennent pas dans la solution.

\subsection{Sensibilité au coût d'une population}

Si l'on effectue une observation d'une matrice de trafic $T=\left[t_{i j}\right]$ $c_{r}=\frac{1}{N} \sum_{i j} t_{i j} c_{i j}$ est le coût moyen effectivement dépensé en transport par les habitants de la ville.

Il est clair que $c_{r} \geqslant c_{\min }$ par définition et de plus que $c_{r} \leqslant c^{*}$ car sinon cela signifierait que lé coût moyen observé est supérieur au coût obtenu dans le cas où les individus ne tiennent pas compte des coûts dans le choix de leur lieu de travail et de résidence.

Posons :

$$
s=\frac{c^{*}-c_{r}}{c^{*}-c_{\min }}
$$

$s$ est appelé sensibilité au coût de l'ensemble $I$ des habitants de la ville (population active) $s$ est un réel sans dimension compris entre 0 et 1 .

Si $s=0$ la population est insensible au coût. La probabilite de trouver un individu sur le trajet de $Z_{i}$ à $Z_{j}$ vaut $\frac{O_{i} D_{j}}{N^{2}}$ c'est le cas de l'indépendance du choix du domicile et du lieu de travail. 
Si $s=1$ l'affectation à chaque individu d'un domicile et/ou d'un lieu de travail est telle qu'elle minimise les pertes économiques concernant le transport.

Le cas réel est compris entre ces deux extrêmes.

Remarque 1: $\mathrm{Si}$ au lieu de traiter le problème $P_{1}$ avec (9) on le traite avec

$$
\sum_{i j} t_{i j} c_{i j}=N \bar{c}
$$

ce problème n'admet de solution que si $c_{\min } \leqslant \bar{c} \leqslant c_{\max }$ où $c_{\max }$ est la solution du problème de programmation linéaire $P T_{\max }$.

Dans ce cas et avec les notions précédentes on aurait si :

$$
\begin{array}{ll}
c_{\max } \geqslant \bar{c} \geqslant c^{*} & s=\frac{c^{*}-c_{r}}{c^{*}-c_{\max }} \quad-1 \leqslant s \leqslant 0 \\
c^{*} \geqslant \bar{c} \geqslant c_{\min } & s=\frac{c^{*}-c_{r}}{c^{*}-c_{\min }} \quad 0 \leqslant s \leqslant 1
\end{array}
$$

Figure 2.

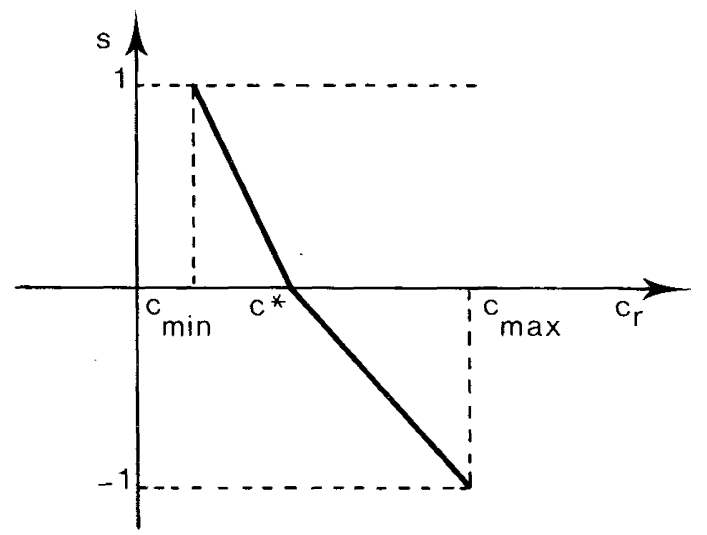

La présentation précédente est justifiée par le fait que dans les exemples étudiés aucune ville ne présente de $s$ négatif (et donc de $\beta$ négatif).

D'autre part, ce qui précède est évidemment applicable à des problèmes de localisation.

REMARQue 2: La sensibilité $s$ est mesurable, c'est-à-dire qu'elle peut être calculée directement à partir d'observations et indépendamment de tout modèle.

Soit $t_{i j}$ une matrice observée de trafic, la matrice $c_{i j}$ est supposée connue. On calcule

$$
O_{i}=\sum_{j} t_{i j} \quad D_{j}=\sum_{i} t_{i j} \quad N=\sum_{i} O_{i}
$$


On résoud le problème $P T_{\min }$ pour les $O_{i}$ et les $D_{j}$ ce qui donne $c_{\min }$.

Cette résolution peut par exemple s'effectuer par l'excellente méthode primale-duale exposée entre autres dans Ford-Fulkerson (1967).

On calcule

$$
c^{*}=\frac{1}{N} \sum_{i j} \frac{O_{i} D_{j}}{N} c_{i j} \quad \text { et } \quad c_{r}=\frac{1}{N} \sum_{i j} t_{i j} c_{i j}
$$

alors $s=\frac{c^{*}-c_{r}}{c^{*}-c_{\min }}$ est une estimation de la sensibilité (il ne s'agit que d'une estimation car les $t_{i j}$ observés ne sont pas forcément à l'équilibre statistique).

REMARQue 3: La sensibilité est une notion relative. Une agglomération étant donnée, envisageons la création d'une nouvelle zone d'emplois très éloignée du centre (le système restant fermé, on supposera la disparition d'un nombre égal d'emplois dans les quartiers centraux) il est clair que les quantités $c_{r}, c^{*}$ et $c_{\min }$ augmentent.

Étant donnée la définition de la sensibilité, on doit s'attendre à ce que $s$ varie peu. Ce fait est à tester. Sa confirmation impliquerait que $s$ est indépendante de la structuration de l'agglomération (localisations relatives des emplois et des résidences) et caractérise donc cette notion difficilement préhensible de comportement des habitants face aux coûts de transport.

\subsection{Nouvelle formulation du problème}

Pour la planification des transports urbains le problème est en général de prévoir les modifications du trafic $T$ sous l'influence d'une modification des coûts $c_{i j}$ et/ou des marges $O_{i}$ et $D_{j}$. On se donne la sensibilité $s$; ceci permet de résoudre le problème suivant :

$P \quad \mid c_{i j}$ et $s$ étant donnés, trouver la matrice de trafic la plus probable dont les marges sont $O_{i}$ et $D_{j}$.

Résolution de $P$ : On résoud: $P T_{\min }$ d'où $c_{\min }$ soit alors :

et

$$
c^{*}=\frac{1}{N} \sum_{i j} \frac{O_{i} D_{j}}{N} c_{i j}
$$

$$
\bar{c}=(1-s) c^{*}+s c_{\min }
$$

et on résoud $S_{1}$ avec cette valeur de $\bar{c}$.

\subsection{4. Étude de la matrice de coût}

3.141. Transformation $n^{\circ} 1$

Le problème $P$ est inchangé si l'on transforme $c_{i j}$ en $\alpha c_{i j}$, $\alpha>0$. En effet, $\bar{c}$ est changé en $\alpha \bar{c}$ et $S_{1}$ est invariant. Ainsi tout changement d'échelle des coûts laisse invariant le trafic. 
3.142. Transformation $n^{\circ} 2$

Le problème $P$ est inchangé si l'on transforme $c_{i j}$ en $c_{i j}+p_{i}+q_{j}$ pour tout $p_{i}$ et $q_{j}$.

- Posons $c_{i j}^{\prime}=c_{i j}+p_{i}+q_{j}$.

Considérons $P T_{\min }$ pour $c_{i j}^{\prime}$

$$
P T_{\min } \mid \begin{gathered}
\overline{t_{i j}^{\prime}} \geqslant 0 \quad \sum_{j} \overline{t_{i j}^{\prime}}=O_{i} \quad \sum_{i} \overline{t_{i j}^{\prime}}=D_{j} \\
\sum_{i j} \overline{t_{i j}^{\prime}} c_{i j}^{\prime}=\sum_{i j} \overline{t_{i j}^{\prime}} c_{i j}+\sum_{i} p_{i} \sum_{j} \overline{t_{i j}^{\prime}}+\sum_{j} q_{j} \sum_{i} \overline{t_{i j}^{\prime}} \\
=\sum_{i j} \overline{t_{i j}^{\prime}} c_{i j}+\sum_{i}\left(p_{i} O_{i}+q_{i} D_{i}\right)
\end{gathered}
$$

$P T_{\min }$ pour $c_{i j}^{\prime}$ conduit alors à la même solution que $P T_{\min }$ pour $c_{i j}$ car la quantité à minimiser ne diffère dans les deux cas que par une constante additive. On a alors :

$$
c_{\min }^{\prime}=c_{\min }+\frac{1}{N} \sum_{i}\left(p_{i} O_{i}+q_{i} D_{i}\right)
$$

- D'autre part:

d'où :

$$
c^{* \prime}=\frac{1}{N} \sum_{i j} \frac{O_{i} D_{j}}{N}\left(c_{i j}+p_{i}+q_{j}\right)=c^{*}+\frac{1}{N} \sum_{i}\left(p_{i} O_{i}+q_{i} D_{i}\right) / N
$$

$$
\begin{gathered}
\bar{c}^{\prime}=c^{* \prime}-s\left(c^{* \prime}-c_{\min }^{\prime}\right)=c^{*}-s\left(c^{*}-c_{\min }\right)+\frac{1}{N} \sum_{i}\left(p_{i} O_{i}+q_{i} D_{i}\right) \\
\bar{c}^{\prime}=\bar{c}+\frac{1}{N} \sum_{i}\left(p_{i} O_{i}+q_{i} D_{i}\right)
\end{gathered}
$$

et $S_{1}$ s'écrit :

$$
\mid \begin{aligned}
& t_{i j}^{\prime}=a_{i} b_{j} \mathrm{e}^{-\beta\left(c_{i j}+p_{i}+q_{j}\right)} \\
& \sum_{j} t_{i j}^{\prime}=O_{i} \quad \sum_{i} t_{i j}^{\prime}=D_{j} \\
& \sum_{i j} t_{i j}^{\prime}\left(c_{i j}+p_{i}+q_{j}\right)=N \bar{c}+\sum_{i}\left(p_{i} O_{i}+q_{i} D_{i}\right)
\end{aligned}
$$

posons $A_{i}=a_{i} e^{-\beta p_{i}} \quad$ et $\quad B_{j}=b_{j} e^{-\beta q_{j}}$

on a :

$$
\mid \begin{aligned}
& t_{i j}^{\prime}=A_{i} B_{j} \mathrm{e}^{-\beta c_{i j}} \\
& \sum_{i j} t_{i j}^{\prime}=O_{i} \quad \sum_{i} t_{i j}^{\prime}=D_{j} \\
& \sum_{i j} t_{i j}^{\prime} c_{i j}=N \bar{c}
\end{aligned}
$$

Système identique à $S_{1}$ donc $t_{i j}^{\prime}=t_{i j}$.

REMARQUE: On peut interpréter $p_{i}$ comme une taxe ou une prime à l'habitation en $Z_{i}$, de même $q_{j}$ pour l'emploi en $Z_{j}$. Le résultat démontré assure l'invariance du trafic; cela est essentiellement dû aux contraintes $C T$ qui imposent les émissions et réceptions des zones. 
3.143. Détermination d'une matrice de coût connaissant une matrice de trafic observée

Soit $t_{i j}$ cette matrice; on se place toujours dans l'hypothèse du modèle à une seule matrice de coût. Alors existent $a_{i}, b_{j}, \beta$ et une matrice de coût $c_{i j}^{\prime}$ tels que :

$$
t_{i j}=a_{i} b_{j} e^{-\beta c_{i j}^{i j}}
$$

En utilisant les transformations $\mathrm{n}^{\circ} 1$ et $2, c_{i j}^{\prime}$ peut être remplacée par la matrice de coût équivalente

et

$$
\begin{aligned}
& c_{i j}=\beta c_{i j}^{\prime}+p_{i}+q_{j} \\
& t_{i j}=a_{i} b_{j} e^{-c_{i j}+p_{i}+q_{j}}
\end{aligned}
$$

posons $p_{i}=-\log a_{i}, q_{j}=-\log b_{j}$

on a: $\quad t_{i j}=e^{-c_{i j}}$

d'où une matrice de coût possible, c'est-à-dire telle que le trafic le plus probable associé soit $t_{i j}$ :

$$
c_{i j}=-\log t_{i j}
$$

On peut d'ailleurs faire sur cette matrice toutes transformations 1 et 2 et notamment l'ajuster à une matrice de distances.

3.144. Quelques tests

La carte jointe représente un découpage de l'agglomération lyonnaise en 6 zones.

La figure 3 est le tableau des distances moyennes $D=\left[d_{i j}\right]$ à vol d'oiseau inter zones et intra zone mesurées sur la carte.

La figure 4 est le tableau des coûts $C=\left[c_{i j}\right]$ obtenu selon la méthode exposée en 3.143 à partir d'une enquête effectuée à Lyon en 1962. Afin de comparer $C$ au tableau $D$ on a de plus effectué les transformations vues en 3.141 et 3.142 de sorte que si

$$
c_{i j}=\alpha \log t_{i j}+p_{i}+q_{j}
$$

les $\alpha, p_{i}, q_{j}$ sont solution du problème :

$$
\min _{\alpha, p_{i}, q_{j}} \sum_{i j}\left(c_{i j}-d_{i j}\right)^{2}
$$

On notera l'assez bonne adéquation des résultats et notamment la quasisymétrie de $C$. L'écart le plus notable est observé pour $C_{23}$. Cela est facilement explicable par la présence du Rhône entre les zones 2 et 3 et l'existence d'un seul pont sur cette frontière, situé en $*$ sur la carte. Ceci est d'autant plus remarquable que les hypothèses sous-jacentes à ce calcul sont brutales : tous modes et motifs de déplacement confondus et uniformité du comportement de la population. 


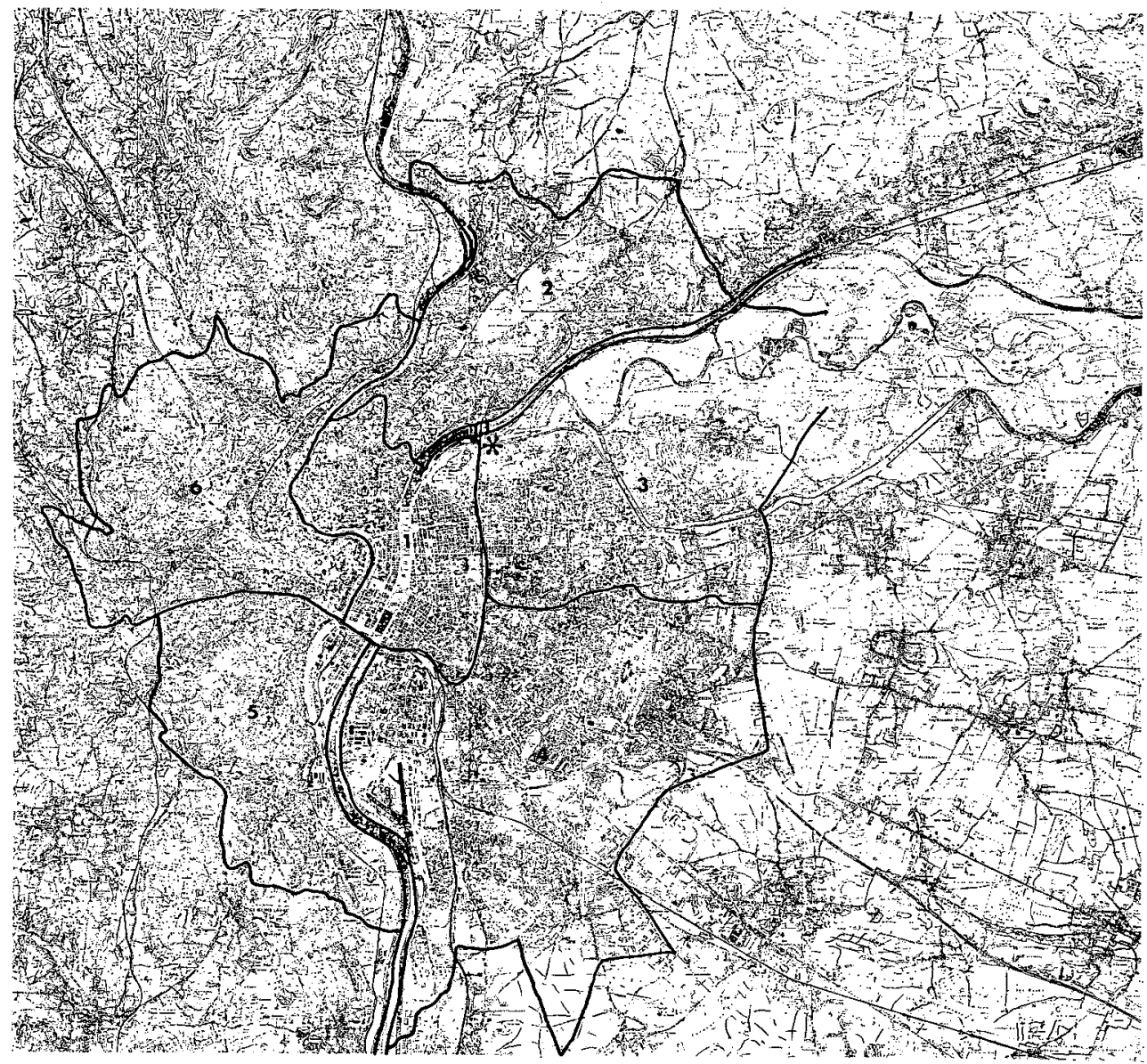

vol. $11, \mathrm{n}^{\circ} 3$, août 1977 


\begin{tabular}{|c|c|c|c|c|c|c|}
\hline & 1 & 2 & 3 & 4 & 5 & 6 \\
\hline 1 & 37 & 71 & 71 & 86 & 60 & 61 \\
\hline 2 & 71 & 61 & 72 & 142 & 95 & 121 \\
\hline 3 & 71 & 72 & 57 & 107 & 125 & 125 \\
\hline 4 & 86 & 142 & 107 & 71 & 112 & 78 \\
\hline 5 & 60 & 95 & 125 & 112 & 59 & 83 \\
\hline 6 & 61 & 121 & 125 & 78 & 83 & 51 \\
\hline
\end{tabular}

Figure 3.

Tableau des distances moyennes à vol d'oiseau.

\begin{tabular}{|c|c|c|c|c|c|c|}
\hline & 1 & 2 & 3 & 4 & 5 & 6 \\
\hline 1 & 28.4 & 70.2 & 70.9 & 77.5 & 68.4 & 70.7 \\
\hline 2 & 73.7 & 47.5 & 108.2 & 122.1 & 99.0 & 111.4 \\
\hline 3 & 71.8 & 103.7 & 65.5 & 103.1 & 106.6 & 106.3 \\
\hline 4 & 77.2 & 122.7 & 101.5 & 82.1 & 112.2 & 100.3 \\
\hline 5 & 63.1 & 106.9 & 103.9 & 110.2 & 53.4 & 91.9 \\
\hline 6 & 63.8 & 110.9 & 107.3 & 101.0 & 94.4 & 38.4 \\
\hline
\end{tabular}

Figure 4.

Tableau des coûts

\begin{tabular}{|c|c|c|c|}
\hline & LYON. & MÜLHOUSE & $A \mathrm{EN}$ \\
\hline $\begin{array}{l}\text { taille de la fopuiation } \\
\text { considérée }\end{array}$ & 343272 & 225469 & 216893 \\
\hline Sensibilité $S$ & 0.57 & 0.33 & 0.26 \\
\hline
\end{tabular}

Figure 5.

Sensibilités.

La figure 5 donne enfin des valeurs de la sensibilité au coût $s$ pour les villes de Lyon, Mulhouse et Caen. Les matrices de coût sont obtenues comme précédemment à partir de l'observation des matrices de trafic.

On retrouve un caractère spécifique des Lyonnais mis en évidence par des études socio-économiques: rechercher des trajets domicile-travail assez courts; ce qui se traduit ici par une grande sensibilité au coût (voir remarque 3 de 3.12).

R.A.I.R.O. Recherche Opérationnelle/Operations Research 
3.15. Connaissant une matrice de trafic observée trouver la nouvelle matrice de trafic lorsque seules les marges sont modifiées

Ce problème diffère de celui posé en 3.13 par le fait que les $c_{i j}$ sont inconnus. Il s'agit en fait du problème de la prévision d'un tableau dont on a prédéterminé les marges. Il existe plusieurs méthodes plus ou moins empiriques pour résoudre ce problème, voir par exemple Fages (1971).

Dans ce cas particulier on peut procéder ainsi :

- Calculer la matrice de coût par (14), la rendre positive par une transformation 2. Cette matrice sera conservée car seules les marges sont modifiées.

- Calculer la sensibilité $s$ pour les coûts ainsi calculés (cf. 3.12, remarque 2 ).

- Traiter le problème $P$ avec les coûts calculés, la sensibilité $s$ et les nouvelles marges $O_{i}$ et $D_{j}$.

Cette façon de procéder suppose l'invariance de $s$. En fait cela revient à sủpposer que les individus ont gardé le même comportement face aux coûts.

\subsection{Modèle de trafic à plusieurs matrices de coûts}

Supposons maintenant qu'il y ait dans l'agglomération considérée $m$ modes de transport, on se donne donc $m$ matrices de coût $\left[c_{i j}^{k}\right], k=1, m$.

Un trafic $T$ est défini par les $m$ matrices $\left[t_{i j}^{k}\right]$ où $t_{i j}^{k}$ est le nombre d'individus se rendant de $Z_{i}$ à $Z_{j}$ par le mode $k$.

Si $\sum_{i j} t_{i j}^{k}=N_{k}$ avec $\sum_{k} N_{k}=N$, on définit $H$ par

$$
H=\log \left(\prod_{k} \frac{N_{k} !}{\prod_{i j}\left(t_{i j}^{k} !\right)}\right)
$$

En supposant toujours que les individus ont le même comportement relativement aux coûts, C.T. et la condition de comportement s'écrivent

$$
\mid \begin{aligned}
& t_{i j}^{k} \geqslant 0 \quad \quad \sum_{i j} t_{i j}^{k}=N_{k} \\
& \sum_{j k} t_{i j}^{k}=O_{i} \quad \sum_{i k} t_{i j}^{k}=D_{j} \quad \text { C.T. } \\
& \sum_{i j k} t_{i j}^{k} c_{i j}^{k} \leqslant N \bar{c}
\end{aligned}
$$

3.21. Problème aux $N_{k}$ fixés

Supposons les $N_{k}$ donnés, $H$ est fonction strictement concave des $t_{i j}^{k}$ après prolongement aux réels comme en 1.12 
posons

$$
t_{i j_{k}}^{k *}=\frac{O_{i} D_{j}}{N^{2}} N_{k}
$$

et

$$
c^{*}=\frac{1}{N} \sum_{i j k} t_{i j}^{k *} c_{i j}^{k}
$$

Soit $\bar{t}_{i j}^{k}$ solution de

$$
P T_{\min }^{m} \mid \begin{aligned}
& \operatorname{Min} \sum_{i j k} \bar{t}_{i j}^{k} c_{i j}^{k} \\
& \text { Sous les contraintes C.T. }
\end{aligned}
$$

posons

$$
c_{\min }=\frac{1}{N} \sum_{i j k} \bar{t}_{i j}^{k} c_{i j}^{k}
$$

En utilisant la méthode des multiplicateurs de Lagrange on aura pour $t_{i j}^{k}$ solution de $H$ maximum sous C.T. et (15).

$$
\text { Si } \quad \bar{c} \geqslant c^{*} \quad t_{i j}^{k}=\frac{O_{i} D_{j}}{N^{2}} N_{k}
$$

Si $c_{\min }<\bar{c}<c^{*} t_{i, j}^{k}$ est solution du système

$$
\mid \begin{aligned}
& t_{i j}^{k}=a_{i} b_{j} \gamma_{k} \mathrm{e}^{-\beta c_{i j}^{k}} \\
& \sum_{i j} t_{i j}^{k}=N_{k} \\
& \sum_{j k} t_{i j}^{k}=O_{i} \quad, \quad \sum_{i k} t_{i j}^{k}=D_{j} \\
& \sum_{i j k} t_{i j}^{k} c_{i j}^{k}=N \bar{c}
\end{aligned}
$$

Si $\bar{c}=c_{\min } t_{i j}^{k}=$ une solution de $P T_{\min }$ et si $\bar{c}<c_{\min }$ pas de solution.

On peut à nouveau introduire la notion de sensibilité aux coûts qui sera ici la même pour tous les modes de transport. D'autre part les remarques de 3.12 restent valables.

\subsection{Problème aux $N_{k}$ inconnus}

Ce problème est important en pratique car il permet de prévoir l'incidence d'un nouveau mode de transport, par exemple la création d'un métro, sur le trafic et de traiter la question du choix du mode de transport par les usagers. Le problème est posé dans les mêmes termes que précédemment mais les $N_{k}$ sont inconnus. 
On est alors amené à résoudre le système

$$
\mid \begin{aligned}
& t_{i j}^{k}=a_{i} b_{j} \gamma_{k} \mathrm{e}^{-\beta c_{i j}^{k}} \\
& \sum_{k} \gamma_{k}=1 \\
& \sum_{i j} t_{i j}^{k}=N \gamma_{k}, \\
& \sum_{j k}^{i j} t_{i j}^{k}=O_{i}, \quad \sum_{i k} t_{i j}^{k}=D_{j} \\
& \sum_{i j k} t_{i j}^{k} c_{i j}^{k}=N \bar{c}
\end{aligned}
$$

aux inconnues $a_{i}, b_{j}, \gamma_{k}$ et $\beta$.

\subsection{Modèle de trafic à plusieurs comportements}

$\mathrm{Au}$ lieu de désagréger les modes de transport on peut désagréger l'ensemble des individus $I$. Supposons donc qu'il y ait $m$ catégories d'individus et une contrainte de comportement par catégorie. On ne se donne qu'une seule matrice de coût pour simplifier mais le problème peut être traité avec plusieurs modes de transport.

Pour chaque catégorie $k, k=1, m$, on cherche les $t_{i j}^{k}$ avec $\sum_{i j} t_{i j}^{k}=M_{k}$, les $M_{k}$ sont connus, car on s'est donné une stratification de la population.

Le trafic le plus probable est solution de

$$
\max _{t_{i j}^{k}} H=\log \left(\prod_{k} \frac{M_{k} !}{\prod_{i j}\left(t_{i j}^{k} !\right)}\right)
$$

Sous

$$
\mid \begin{aligned}
& t_{i j}^{k} \geqslant 0 \quad \sum_{i j} t_{i j}^{k}=M_{k} \\
& \sum_{j k} t_{i j}^{k}=O_{i} \quad \sum_{i k} t_{i j}^{k}=D_{j} \quad \text { C.T. } \\
& \sum_{i j} t_{i j}^{k} c_{i j} \leqslant M_{k} \bar{c}_{k} \quad k \text { conditions de comportement }
\end{aligned}
$$

Ce problème est soluble par la méthode des multiplicateurs de Lagrange. Dans ce cas on obtiendra une sensibilité par catégorie d'individus, car il résulte de cette formulation que dans chacune des catégories, les individus ont le même comportement. 


\section{CONCLUSIONS}

Ce qui précède ne contient pas de résultats nouveaux à proprement parler; nous nous sommes attachés à retracer de façon rigoureuse la construction de la distribution la plus probable. Le fait d'utiliser la fonction gamma dans le calcul des dérivées partielles de $H$ assure maintenant que la validité du modèle est toute entière contenue dans l'hypothèse d'équiprobabilité des états de trafic, ce qui distingue nettement cette approche de celle des modèles gravitaires. Ceci est très brutal et de bons ajustements des résultats du modèle aux valeurs observées ne permettent que la formulation suivante: tout se passe comme si l'hypothèse d'équiprobabilité était compatible avec la réalité. On ne peut rien en conclure sur la vérité de l'hypothèse et il est vraisemblable qu'elle représente l'agrégation de phénomènes beaucoup plus fins encore mal connus. Le calcul supposant la connaissance de la valeur moyenne des coûts de transport il serait par ailleurs utile de disposer d'un intervalle de confiance pour celle-ci.

Les transformations (1) et (2) sur la matrice des coûts montrent bien qu'il est difficile d'inférer des $t_{i j}$ observés des conclusions sur la façon dont les habitants perçoivent les coûts. On mentionnera ici à titre d'hypothèse que les modèles de transport du type étudié dans cet article doivent leur bonne adéquation au réel à la répartition relative des résidences et des emplois. C'est dans une étude des localisations que ces modèles peuvent éventuellement trouver une justification.

Nous avons présenté le problème des capacités prédictives du modèle de façon non classique. Les prévisions sont faites usuellement à $\beta$ où $\bar{c}$ constant, ce qui est commode mais peu justifié (il est en particulier remarquable qu'il n'existe pas d'études sur la variation de $\bar{c}$ dans le temps). On peut par contre penser que la notion de sensibilité que nous avons définie est susceptible de cerner le problème du comportement des habitants face aux coûts de façon relativement indépendante de la morphologie des localisations et du réseau de transport. Le modèle permet par ailleurs la comparaison de plans alternatifs de localisation des emplois et des résidences.

Nous nous proposons dans la suite de ce travail de faire un certain nombre d'expériences sur des données réelles et d'envisager certaines questions théoriques comme le choix judicieux du nombre et de la taille des zones ainsi que l'étude des fluctuations des $t_{i j}$ autour de l'équilibre statistique seul considéré ici. 


\section{BIBLIOGRAPHIE}

M. BACHARACH, Estimating nonnegatives matrices from marginal data, International Economic Review, Vol. 6, n 3,.1965.

T. Broadbent, Zone Size and Singly-Constrained Interaction models, Working note $\mathrm{n}^{\circ}$ 132, Centre for Environmental Studies, London, 1969.

R. Bussiere, Modèles Mathématiques de répartition des populations urbaines, C.R.U., Paris, 1972.

A. Evans. The calibration of trip distribution models with exponential or similar cost functions, Transportation research, 5, 1971, p. 15-38.

S. Evans, A relationship between the gravity model for trip distribution and the transportation problem in linear programming, Transportation research, 7, 1973, p. 39-61.

R. FAGES, Tableaux à marges fixées. Transformations RAS et DSM, Publications Économétriques, Vol. IV, Fasc. 1, 1971, p. 240-253.

L. Ford et D. FulKerson, Flots dans les graphes, Gauthier-Villars, Paris, 1967.

E. JAYnEs, Prior probabilities, IEEE Trans. Systems Science and Cybernetics, SSC-4, 1968, p. 227-241.

D. LINDLEY, Bayesian statistics, a review, SIAM, Philadelphia, 1972.

L. MARCH, Models, modes and mores, LUBFS Conference Proc., 3, 1975, p. 301-310.

M. Tribus, Rational descriptions, decisions and designs, Pergamon Press, Oxford, 1969.

A. Wilson, Entropy in urban and regional modelling, Pion, London, 1970.

A. WiLson, Urban and regional models in geography and planning, Wiley, London, 1974. 